\title{
Transporter Gene Expression in Human Head and Neck Squamous Cell Carcinoma and Associated Epigenetic Regulatory Mechanisms
}

Oliver Zolk, ${ }^{*}$ Rebecca Schnepf, ${ }^{*}$ Marc Muschler, ${ }^{\dagger}$ Martin F. Fromm, ${ }^{*}$ Olaf Wendler, ${ }^{\ddagger}$ Maximilian Traxdorf, ${ }^{\ddagger}$ Heinrich Iro, ${ }^{\ddagger}$ and Johannes Zenk ${ }^{\ddagger}$

From the Institute of Experimental and Clinical Pharmacology and Toxicology* and the Department of Otorhinolaryngology, Head and Neck Surgery, ${ }^{\ddagger}$ Friedrich-Alexander-Universität Erlangen-Nürnberg, Erlangen; and the Department of Psychiatry, ${ }^{\dagger}$ Center for Addiction Research, Social Psychiatry and Psychotherapy, Hannover Medical School, Hannover, Germany

Accepted for publication September 13, 2012.

Address correspondence to Oliver Zolk, M.D., Institute of Experimental and Clinical Pharmacology and Toxicology, Friedrich-Alexander-Universität Erlangen-Nürnberg, Fahrstrasse 17, 91054 Erlangen, Germany. E-mail: Zolk@ pharmakologie.med. uni-erlangen.de.

\begin{abstract}
Expression levels of membrane transporters may affect the disposition, and thereby treatment efficacy, of anticancer drugs in human head and neck squamous cell carcinoma (HNSCC). Herein, we analyzed the gene expression profile of membrane transporters in HNSCC. In addition, we evaluated the mechanisms of transporter regulation in HNSCC and focused on the role of the nuclear pregnane $X$ receptor (or NR1I2) and epigenetic mechanisms. Real-time RT-PCR revealed a significantly increased mRNA expression of membrane transporters SLCO1A2 and SLCO1B3 and a significantly decreased expression of transporters SLCO2B1, SLCO2A1, and $A B C C 3$ in human HNSCC tumors compared with adjacent normal mucosa. An association between SLCO2B1 mRNA levels in tumors and 5-year survival of patients with HNSCC was observed $\left(\chi^{2}=6.59, P=0.010\right)$. Bisulfite sequencing revealed that promoter $\mathrm{CpG}$ islands of $A B C C 3$ and $S \angle C O 2 A 1$ were not hypermethylated, indicating that these genes were not epigenetically silenced in HNSCC tumors. In HNSCC-derived cell lines, transcript expression of transporters (eg, ABCC3 or SLC02A1; $P<0.001$ for both) and NR1I2 $(P<0.001)$ was markedly induced by the DNA methyltransferase inhibitor, decitabine. Cotreatment with the prototypical pregnane $X$ receptor activator, rifampicin, significantly reversed decitabine-induced $A B C C 3$ and SLCO2A1 expression. In summary, the expression of drug transporters $i$ ) is markedly changed in HNSCC tumor tissues compared with normal mucosa, ii) might be predictive of the outcome of patients with HNSCC, and iii) is affected by novel epigenetic therapies and is further modulated by nuclear receptor-mediated mechanisms. (Am J Pathol 2013, 182: 234-243; http://dx.doi.org/10.1016/j.ajpath.2012.09.008)
\end{abstract}

The interindividual variability in drug response and the emergence of adverse drug reactions are the main causes of treatment failure in cancer therapy. ${ }^{1}$ Recently, membrane transporters have been recognized as an important determinant of drug disposition, thereby affecting chemosensitivity and drug resistance. Moreover, expression levels of transporters have been associated with clinical outcomes in patients with cancer. ${ }^{1}$

Two major superfamilies of membrane transporters that influence drug pharmacokinetics are the ATP-binding cassette $(\mathrm{ABC})$ transporters and the transporters belonging to the solute carrier (SLC) family, such as the organic anion transporting polypeptides (OATPs; encoded by the SLCO genes) or the SLC22 organic cation/anion/zwitterion transporters. $^{2}$ The ABC transporters are responsible for the export of diverse substrates, including anticancer drugs, from the cell against a concentration gradient, with ATP hydrolysis providing the driving force. ${ }^{3,4}$ The extrusion of anticancer drugs from the tumor cells often results in multidrug resistance of tumors. ${ }^{3}$

The OATPs are involved in cellular influx mechanisms. Known substrates of the OATP family include endogenous compounds and a variety of pharmaceuticals, including

Supported by the Johannes and Frieda Marohn Foundation 
anticancer drugs. ${ }^{5-7}$ The OATPs expressed in the liver have contributed to systemic variation in anticancer drug treatments. ${ }^{5,6}$ Moreover, OATPs expressed in tumors may be implicated in cellular uptake of anticancer drugs, thereby increasing the chemosensitivity of tumor cells. ${ }^{1}$

The profile and the mechanisms of transporter expression in human head and neck squamous cell carcinoma (HNSCC), one of the most frequent cancers, with an incidence of $>200,000$ new cases worldwide annually, ${ }^{8}$ have not been studied. Therefore, the first aim of our study was to identify transporters that are differentially expressed in HNSCC compared with adjacent normal control tissue. In addition, the expression of transporter genes was correlated with disease stage and 5-year survival in a small-scale preliminary study.

The second aim of our study was to evaluate mechanisms by which the transporters are regulated in HNSCC. Given that the disruption of the epigenome is a fundamental mechanism in cancer and that the reversal of epigenetic changes represents a potential target of novel treatments in cancer, we focused on epigenetic mechanisms (ie, changes in transporter gene expression that are mediated by DNA methylation). ${ }^{9}$ DNA methylation of the $\mathrm{CpG}$ islands within the transporter gene promoters can cause repression of gene expression either directly, by the steric hindrance of methyl-CpG interaction with transcription factors, or indirectly, through the recruitment of methyl-binding proteins that associate with chromatin-modifying proteins to produce a complementary repressive heterochromatin environment. ${ }^{10,11}$ Although gene silencing via aberrant DNA promoter hypermethylation explains some aspects in the tumorigenesis of HNSCC, ${ }^{12}$ little is known about the contribution of epigenetics to the regulation of drug transporter expression. $^{11}$

Most recent studies suggest that transporter genes, such as $A B C B 1$ (P-glycoprotein), are targets of the transcriptional activator, pregnane $\mathrm{X}$ receptor (PXR; alias NRII2) ${ }^{13}$ Several anticancer drugs (eg, taxanes and cisplatin) can activate PXR. ${ }^{14,15}$ Moreover, promoter methylation is involved at least in the regulation of PXR in cancer cells, ${ }^{16,17}$ raising the possibility of interactions between epigenetic and nuclear receptor-mediated mechanisms in the regulation of transporter expression in HNSCC.

Therefore, the role of PXR and the epigenetic mechanism of transcriptional regulation of selected SLC and ABC transporters were evaluated in the present study by analyzing the response of human HNSCC cell lines (UMSCC-1 and SCC-15) to the DNA methyltransferase inhibitor, decitabine (5-aza-2'-deoxycytidine), and to the PXR activator, rifampicin, as single or combined treatments. Moreover, the effects of NR1I2/PXR knockdown by lentivirus-mediated short-hairpin RNA (shRNA) interference on basal and decitabine-modified transporter expression were evaluated. Promoter $\mathrm{CpG}$ methylation was investigated using bisulfite sequencing.

\section{Materials and Methods}

\section{Tissue Samples}

Human HNSCC tissues and adjacent normal mucosa tissues from patients with HNSCC were attained from the Department of Otorhinolaryngology, Head and Neck Surgery, University of Erlangen-Nuremberg (Erlangen, Germany). A histopathological evaluation was performed on all samples for verification. Patients gave informed consent, and the study was approved by the ethics committee of the University of Erlangen-Nuremberg. None of the patients had received neoadjuvant chemotherapy. The primary tumor and paired macroscopically healthy tissue from the excision border were obtained at surgery. The samples with a normal phenotype were located distant from the macroscopic tumor edge, as judged by the surgeon. Tumor material and adjacent normal mucosa were collected, snap frozen in liquid nitrogen, and stored at $-80^{\circ} \mathrm{C}$ before use. For quality control, cryosections were investigated by a pathologist. Patient details are shown in Table 1.

\section{Cell Culture and Drug Treatments}

Human UMSCC-1 cells, derived from an oral cavity carcinoma, were obtained from the Department of Otolaryngology/Head and Neck Surgery, University of Michigan School of Medicine (Ann Arbor, MI). ${ }^{18}$ The SCC-15 cell line, derived from a squamous cell carcinoma of the tongue, was obtained from ATCC (Manassas, VA; reference CRL-1623). The cell lines were recently characterized and validated by short tandem repeat profiling in two independent studies. ${ }^{19,20}$ To generate stable UMSCC cells with reduced NRII2 mRNA expression, Mission NRII2 shRNA lentiviruses (NM_003889; clones TRCN00000211619, TRCN00000211621, TRCN00000211622, and TRCN00000211623; Sigma-Aldrich, St. Louis, MO), with a titer of $>10^{6}$ transforming units $/ \mathrm{mL}$, were used to infect UMSCC-1 cells. Subsequently, stable clones were selected using puromycin $(2 \mu \mathrm{g} / \mathrm{mL})$. Control UMSCC cells were infected with nonsilencing control lentiviral particles (SHC002V; Sigma-Aldrich). UMSCC cells were maintained in Dulbecco's modified Eagle's medium and Ham's F12 with GlutaMAX (Invitrogen, Carlsbad, CA), supplemented with $10 \%$ fetal bovine serum (Invitrogen), $100 \mathrm{U} /$ $\mathrm{mL}$ penicillin (Invitrogen), $100 \mu \mathrm{g} / \mathrm{mL}$ streptomycin (Invitrogen), and $1 \mathrm{mmol} / \mathrm{L}$ sodium pyruvate (Invitrogen). SCC15 cells were maintained in Dulbecco's modified Eagle's medium and Ham's F12 medium containing $1.2 \mathrm{~g} / \mathrm{L}$ sodium bicarbonate, $2.5 \mathrm{mmol} / \mathrm{L}$ L-glutamine, $15 \mathrm{mmol} / \mathrm{L}$ HEPES, and $0.5 \mathrm{mmol} / \mathrm{L}$ sodium pyruvate, supplemented with 100 $\mathrm{U} / \mathrm{mL}$ penicillin (Invitrogen), $100 \mu \mathrm{g} / \mathrm{mL}$ streptomycin (Invitrogen), $400 \mathrm{ng} / \mathrm{mL}$ hydrocortisone, and $10 \%$ fetal bovine serum. For drug treatments, cells were plated on 60-mm dishes and cultured to $75 \%$ confluence. Drugs (all from Sigma-Aldrich) were dissolved in dimethyl sulfoxide 
Table 1 Characteristics of Patients with HNSCC

\begin{tabular}{|c|c|c|c|c|c|}
\hline $\begin{array}{l}\text { Patient } \\
\text { no. }\end{array}$ & $\begin{array}{l}\text { Age } \\
\text { (years) }\end{array}$ & Sex & Localization & $\begin{array}{l}\text { ICD-10 } \\
\text { code }\end{array}$ & pT class \\
\hline 1 & 57 & Male & Pharynx & $\mathrm{C} 12$ & $\mathrm{~T} 2$ \\
\hline 2 & 38 & Male & Glottis & C32.0 & $\mathrm{T} 3$ \\
\hline 3 & 68 & Female & Larynx & C32.0 & $\mathrm{T} 4$ \\
\hline 4 & 65 & Male & Larynx & C32.2 & $\mathrm{Tx}$ \\
\hline 5 & 58 & Male & Larynx & C 32.0 & $\mathrm{~T} 2$ \\
\hline 6 & 46 & Female & Larynx & C32.0 & T4 \\
\hline 7 & 55 & Male & Larynx & C32.1 & $\mathrm{T} 2$ \\
\hline 8 & 77 & Male & Floor of mouth & Co4.8 & $\mathrm{T} 2$ \\
\hline 9 & 74 & Female & Tonsil & Co9.9 & T3 \\
\hline 10 & 51 & Male & Larynx & C32.1 & $\mathrm{T} 3$ \\
\hline 11 & 65 & Female & Larynx & C32.0 & $\mathrm{T} 3$ \\
\hline 12 & 44 & Male & Tonsil & C05.1 & $\mathrm{T} 1$ \\
\hline 13 & 82 & Male & Tonsil & Co9.9 & $\mathrm{T} 2$ \\
\hline 14 & 63 & Male & Floor of mouth & $\mathrm{CO1}$ & $\mathrm{T} 3$ \\
\hline 15 & 63 & Male & Floor of mouth & $\mathrm{CO1}$ & $\mathrm{T} 1$ \\
\hline 16 & 51 & Male & Larynx & C32.0 & $\mathrm{Tx}$ \\
\hline 17 & 51 & Male & Supraglottis & C32.1 & $\mathrm{T} 2$ \\
\hline 18 & 57 & Female & Oropharynx & $\mathrm{C} 10.3$ & T3 \\
\hline 19 & 61 & Male & Larynx & C32.1 & $\mathrm{T} 1$ \\
\hline 20 & 50 & Male & Larynx & C32.0 & T3 \\
\hline 21 & 57 & Male & Oropharynx & $\mathrm{C} 10.9$ & T4 \\
\hline 22 & 76 & Male & Larynx & C32.0 & T4 \\
\hline 23 & 68 & Male & Larynx & C32.1 & T4 \\
\hline 24 & 80 & Male & Larynx & C32.0 & T4 \\
\hline 25 & 67 & Female & Floor of mouth & C02.1 & $\mathrm{T} 1$ \\
\hline 26 & 44 & Male & Floor of mouth & $\mathrm{C} 04.0$ & $\mathrm{~T} 2$ \\
\hline 27 & 53 & Male & Larynx & C32.0 & T4 \\
\hline 28 & 42 & Male & Larynx & C32.0 & T4 \\
\hline 29 & 57 & Male & Oropharynx & C10.9 & T3 \\
\hline 30 & 57 & Male & Larynx & C32.0 & $\mathrm{T} 1$ \\
\hline 31 & 74 & Male & Larynx & C32.0 & $\mathrm{T} 4$ \\
\hline 32 & 63 & Male & Hypopharynx & $\mathrm{C} 12$ & $\mathrm{~T} 3$ \\
\hline 33 & 52 & Male & Hypopharynx & $\mathrm{C} 12$ & $\mathrm{~T} 2$ \\
\hline 34 & 61 & Male & Larynx & C 32.0 & T4 \\
\hline
\end{tabular}

ICD-10, International Statistical Classification of Diseases and Related Health Problems.

and were used at concentrations as follows: $1 \mu \mathrm{mol} / \mathrm{L}$ decitabine and $25 \mu \mathrm{mol} / \mathrm{L}$ rifampicin. Cells were treated with drugs for 72 hours, with change of medium every 24 hours. Control cells were treated with solvent (dimethyl sulfoxide; $0.1 \%$ to $0.2 \%$ ) only.

\section{RNA Isolation and TaqMan PCR}

Total RNA was isolated from human tissue samples (normal and HNSCC tissue samples from 34 patients) and from UMSCC-1 or SCC-15 cells with TRIzol reagent (Invitrogen), according to the manufacturer's protocol. Before TRIzol was added, frozen human tissue samples were grinded in liquid nitrogen with a mortar and pestle. A total of $5 \mu \mathrm{g}$ of total RNA each was reverse transcribed into cDNA using random primer and Moloney Murine Leukemia Virus Reverse Transcriptase (Promega, Madison, WI) at $37^{\circ} \mathrm{C}$ for 1 hour.
The PCRs $(10 \mu \mathrm{L})$ were set up with $1 \mu \mathrm{L}$ of cDNA (equivalent to $40 \mathrm{ng}$ of input RNA), $5 \mu \mathrm{L}$ of two times QPCR ROX master mix (Thermo Scientific, Rockford, IL), $0.5 \mu \mathrm{L}$ of predesigned fluorogenic TaqMan probe and primer mix (Applied Biosystems, Foster City, CA), and $3.5 \mu \mathrm{L}$ of nuclease-free water. Real-time PCR analysis was performed using the Applied Biosystems ABI PRISM 7900HT Sequence Detection System for 45 cycles, with 3 seconds denaturation at $95^{\circ} \mathrm{C}$ and 30 seconds annealing/extension at $60^{\circ} \mathrm{C}$. Several reference genes were tested, and those showing the highest expression stability were used for normalization. mRNA levels in HNSCC and corresponding normal tissue samples were normalized to $T B T, T U B B$, and $H P R T 1$. For UMSCC-1 and SCC-15 cells, GAPDH and ACTB were used for normalization. To evaluate the amplification efficiency, standard curves were generated using a cDNA standard dilution series. Relative mRNA expression levels were calculated with Sequence Detection System software version 2.1 (Applied Biosystems), which allows correction for differences in amplification efficiency and standardization by reference gene expression.

\section{Bisulfite Sequencing}

Genomic DNA was isolated from decitabine-treated and untreated (solvent controls) UMSCC- 1 cells $(n=3$ each) and from HNSCC and adjacent normal tissues from two patients using the Genomic Tip 20/G extraction kit (Qiagen, Hilden, Germany). Equal amounts of DNA were pooled by treatment group, and DNA $(2 \mu \mathrm{g})$ from each pool was bisulfite converted, according to the manufacturer's instruction, using an EpiTect kit (Qiagen). Primers were designed to anneal to non- $\mathrm{CpG}$ sequences within $\mathrm{CpG}$ islands in $A B C C 3$ and $S L C O 2 A 1$ promoters and, thus, amplify bisulfite-converted genomic DNA regardless of methylation status. For $A B C C 3$, a nested PCR with a second primer pair was performed. Primer sequences are listed in Table 2.

Bisulfite-transformed genomic DNA was amplified twice via PCR using Pfu Turbo $C_{x}$ Hotstart DNA Polymerase (Agilent Technologies, Böblingen, Germany) and Advantage cDNA Polymerase Mix (Clontech, Saint-Germain-enLaye, France). The first round of amplification with Pfu Turbo $\mathrm{C}_{\mathrm{x}}$ Hotstart DNA Polymerase was performed using the following conditions: enzyme activation at $95^{\circ} \mathrm{C}$ for 2 minutes, denaturation at $95^{\circ} \mathrm{C}$ for 30 seconds, annealing at temperatures depending on primer melting temperature for 30 seconds, synthesis at $72^{\circ} \mathrm{C}$ for 1 minute, and final extension at $72^{\circ} \mathrm{C}$ for 10 minutes. The first 10 cycles were run with an annealing temperature of $2^{\circ} \mathrm{C}$, and the subsequent 30 cycles were run with an annealing temperature of $4^{\circ} \mathrm{C}$ lower than primer melting temperature. The second round of amplification was conducted with Advantage cDNA Polymerase Mix. The amplification conditions were as follows: enzyme activation at $94^{\circ} \mathrm{C}$ for 1 minute, denaturation at $94^{\circ} \mathrm{C}$ for 15 seconds, annealing at $52^{\circ} \mathrm{C}$ for 
Table 2 Primers Used for Amplification of Bisulfite-Treated DNA

\begin{tabular}{llcc}
\hline Name & Sequence & Position (relative to TSS) & Fragment length (bp) \\
\hline ABCC3_fw_1 & 5'-GGATTTAGAGGTATTGGGGTAG-3' $^{\prime}$ & 573 \\
ABCC3_rev_1 & 5'-AAAAACCCTCCATCAAAAACC-3' & -63 & 509 \\
ABCC3_fw_2 & 5'-TTAGAGGTATTGGGGTAGG-3' & -59 & 405 \\
ABCC3_rev_2 & $5^{\prime}$-AAACAAAACAAAACAAATCC-3' & 345 & 619 \\
SLC02A1_fw & $5^{\prime}$-GGATTAAGTAATGGGATAGTGGAAG-3' & 1039 & 421 \\
SLC02A1_rev & 5'-CCTAAAACCATTTCCAAACCA-3' & \\
\hline
\end{tabular}

TSS, transcription start site.

30 seconds, synthesis at $68^{\circ} \mathrm{C}$ for 1 minute, and final extension at $68^{\circ} \mathrm{C}$ for 3 minutes. The correct product size and quality of PCR amplicons were controlled by $2 \%$ agarose gel electrophoresis. PCR products were cleaned up using Illustra GFX PCR DNA and Gel Band Purification Kit (GE Healthcare, Freiburg, Germany) to remove any excess nucleotides and primers, and $200 \mathrm{ng}$ each were sequenced directly using the PCR amplification primers. Sequencing data were analyzed using Epigenetic Sequencing Methylation Analysis Software (Epigenomics, Berlin, Germany). The bisulfite sequencing approach allows measurement of DNA methylation with high reproducibility and accuracy. $^{21,22}$

\section{Statistics}

Differences between two groups were tested by the Student's $t$-test for unpaired data. Data were compared with a reference value by the one-sample $t$-test. Differences between more than two groups were tested by one-way analysis of variance and Bonferroni post test. Variables that were not normally distributed (Kolmogorov-Smirnov test) were $\log$ transformed before statistical analysis was performed. The $\chi^{2}$ test was used to analyze associations between transporter expression and $\mathrm{pT}$ class or 5-year survival rates. Logistic regression analysis was used to evaluate the association between transporter expression and 5-year survival rates, with pT class as a covariate. Data were analyzed with SPSS for Windows version 16.0 (SPSS Inc., Chicago, IL) and GraphPad Prism version 5.01 (GraphPad Software, San Diego, CA). $P<0.05$ was required for statistical significance. Data are presented as mean \pm SEM (bar graphs) or as median with lower and upper quartiles and the minimum and maximum of all of the data (box-andwhiskers plots).

\section{Results}

Transporter Expression in Human Normal and HNSCC Tumor Tissues

We generated mRNA expression profiles in human HNSCC tissues of those transporters that have been implicated in cancer development or progression, or in the cellular uptake or efflux of anticancer drugs. Nine members of the solute carrier organic anion transporter family (SLCO1A2, SLCO1B3, SLCO1C1, SLCO2A1, SLCO2B1, SLCO3A1, SLCO4A1, SLCO4C1, and SLCO5A1), two members of the polyspecific solute carrier family 22 (SLC22Al and $S L C 22 A 3)$, and two members of the multidrug and toxin extrusion transporter family (SLC47A1 and SLC47A2) were analyzed. Furthermore, eight $\mathrm{ABC}$ transporters $(A B C B 1$, $A B C C 1, A B C C 2, A B C C 3, A B C C 4, A B C C 5, A B C C 10$, and $A B C G 2$ ) were investigated. Significant differences in expression levels between normal and tumor tissues were observed for 11 transporter genes, as shown in Figure 1A. The expression of SLCO1A2 and SLCO1B3 in tumor samples compared with normal tissues was 9.3-fold $(P<0.001)$ and 4.3 -fold $(P<0.001)$ higher, respectively. In contrast, mRNA concentrations of SLCO4A1, SLCO3A1, SLC47A1, SLC22A3, ABCC3, SLCO2B1, SLCO2A1, $A B C G 2$, and $S L C O 1 C 1$ were significantly decreased by $36 \%$ to $81 \%$ in HNSCC samples compared with the respective control samples.

\section{Transporter Expression versus Disease Stage and 5-Year Survival}

Because previous reports indicated that certain transporters were involved in cancer etiology and progression, we tested whether transporter expression in tumor tissue correlated with tumor stage or survival of patients with HNSCC. A significant association was observed between mRNA expression of SLCO2B1 in HNSCC and improved survival during a 5-year period $(P=0.029)$ (Figure 1B). Comparison of tumor samples with high (upper two quartiles) and low (lower two quartiles) SLCO2B1 mRNA expression (Table 3) revealed a significant association of $S L C O 2 B 1$ expression with the pT class $\left(\chi^{2}=4.394, P=0.036\right)$ and the 5-year survival rate $\left(47 \%\right.$ versus $88 \% ; \chi^{2}=6.585$, $P=0.010)$. The $S L C O 2 B 1$ expression level remained a significant predictor of 5-year survival in our study cohort, even when the $\mathrm{pT}$ class was considered as a covariate in a logistic regression analysis (odds ratio, $6.5 ; 95 \% \mathrm{CI}, 1.1$ to 38.6; $P=0.04)$.

\section{Epigenetic Regulation of Transporter Expression}

Changes in the epigenome, such as aberrant DNA methylation, have been common in cancer. ${ }^{23}$ This raised the 
A

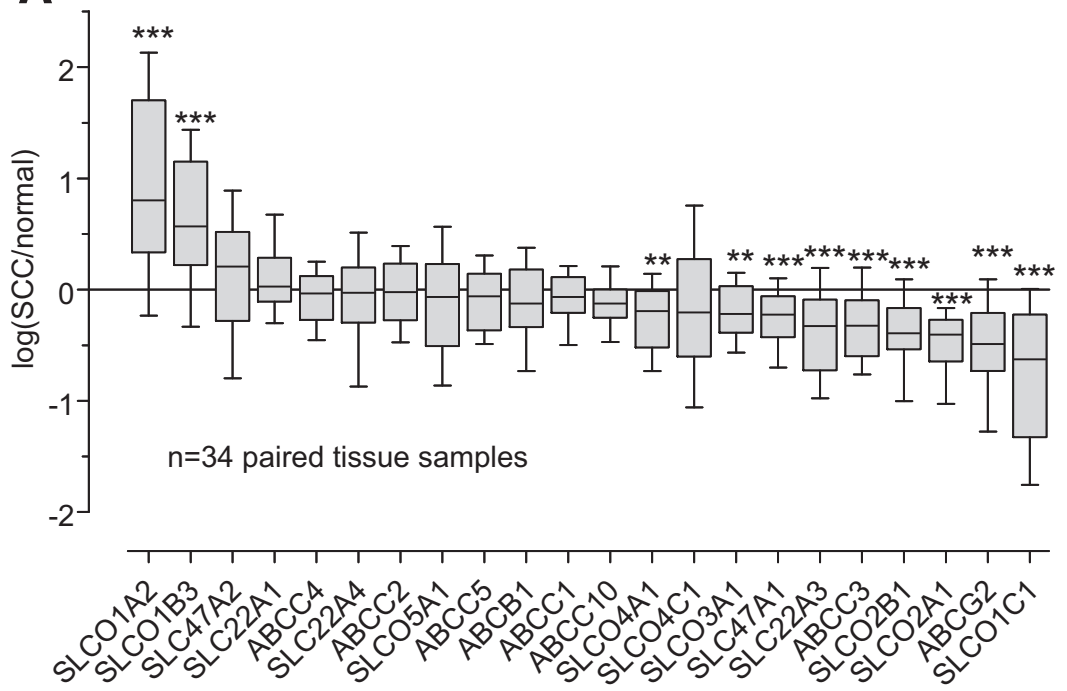

B

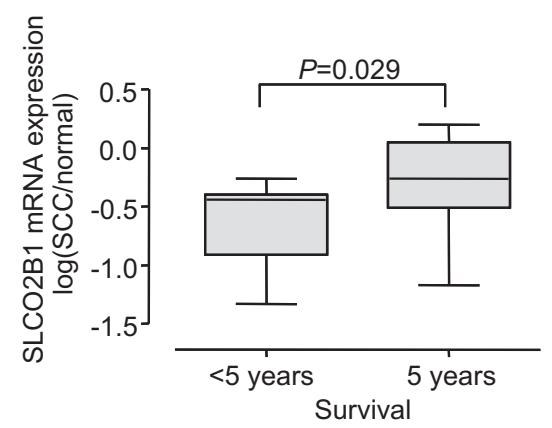

Figure 1 A: Transporter mRNA expression in HNSCC tumor samples compared with normal mucosal tissue from the same patients. Transporter mRNA expression was quantified in paired tumor and normal tissue samples from 34 patients with HNSCC by real-time PCR. The expression was normalized to the reference genes TBT, TUBB, and HPRT1. Data are presented as logarithms of fold change of mRNA expression in tumor versus adjacent normal control tissue. Box-whiskers plots show the median, the lower and the upper quartiles, and the minimum and maximum of all of the data (whiskers). B: Association between SLCO2B1 mRNA expression in HNSCC tumors and 5-year survival. SLCO2B1 mRNA expression in HNSCC tumor samples relative to normal tissues was stratified according to the 5 -year survival. The 5 -year overall survival is $67.6 \%$ (23 of 34 patients). ${ }^{* *} P<0.01,{ }^{* *} P<0.001$.

possibility that expression of transporter genes in HNSCC might be affected by epigenetic mechanisms. To prove this hypothesis, the methylation status of promoter $\mathrm{CpG}$ islands was investigated. Among the transporters that were differentially expressed between normal and HNSCC tissues, $A B C C 3$ and SLCO2Al consisted of promoter $\mathrm{CpG}$ islands (defined as a DNA region of at least $200 \mathrm{bp}$ with a $\mathrm{C}+\mathrm{G}$ content of $50 \%$ and an observed $\mathrm{CpG}$ versus expected $\mathrm{CpG}$ in excess of 0.6). Bisulfite sequencing revealed that promoter $\mathrm{CpG}$ islands of $S L C O 2 A 1$ and $A B C C 3$ were not methylated and, thus, these genes were not epigenetically silenced in HNSCC tissues (Figures 2, 3).

To investigate the consequences of epigenetic modifications on transporter expression, the effects of DNA methyltransferase inhibition or histone deacetylase inhibition on transporter expression were investigated in the human HNSCC cell lines UMSCC-1 and SCC- 15 . We primarily focused on transporters that were implicated in multidrug resistance of cancer cells against cytostatic drugs (ie, multidrug resistance 1: $A B C B 1$; multidrug resistance-associated proteins: $A B C C 1, A B C C 3, A B C C 4$, and $A B C C 5$; and breast cancer resistance protein: $A B C G 2)$. We also investigated transporters whose expression was significantly affected in human HNSCC samples relative to control tissues (SLCO2A1, SLCO2B1, and SLC22A3). In UMSCC-15 cells, DNA methyltransferase inhibition with $1 \mu \mathrm{mol} / \mathrm{L}$ decitabine for 72 hours caused a significant increase in $A B C B 1$ (17.2-fold, $P<0.001)$, ABCC3 (20.8-fold, $P<0.001$ ), ABCG2 (2.8-fold, $P<0.001$ ), SLC22A3 (9.8-fold, $P<$ 0.001), SLCO2A1 (15.8-fold, $P<0.001$ ), and SLCO2B1 (37.3-fold, $P<0.001)$ mRNA expression (Figure 4), whereas gene expression of other transporters, such as $A B C C 1, A B C C 4$, and $A B C C 5$, remained unchanged (data not shown). Qualitatively similar results were obtained in SCC-15 cells (Figure 4).

\section{Role of PXR in the Regulation of Transporter Gene Expression}

The effect of decitabine on transporter mRNA expression might be directly due to a reversal of DNA methylation of CpG-rich sequences in the respective gene promoter. Indeed, decitabine treatment moderately reduced $\mathrm{CpG}$ island methylation in the $S L C O 2 A 1$ promoter by $5.1 \% \pm 1.3 \%(P<0.01)$ (Figure 2). Among the investigated transporters, decitabine treatment caused the most prominent increase in the

Table 3 Association between SLCO2B1 mRNA Expression in Tumors and PT Class and 5-Year Survival Rates in Patients with HNSCC

\begin{tabular}{lcc}
\hline & \multicolumn{2}{c}{ Patients with SLCO2B1 expression in HNSCC } \\
\cline { 2 - 3 } Variable & Weak $(n=17)$ & Strong $(n=17)$ \\
\hline pT class & & $9(60.0)$ \\
T1/2 & $4(23.5)$ & $6(40.0)^{*}$ \\
T3/4 & $13(76.5)$ & $15(88.2)$ \\
Significance & \multicolumn{2}{c}{$\chi^{2}=4.39, P=0.036$} \\
5-Year survival & $8(47.1)$ & $2(11.8)$ \\
Yes & $9(52.9)$ & 0.010 \\
No & \multicolumn{2}{c}{$\chi^{2}=6.59, P=0.9$} \\
Significance &
\end{tabular}

* Two patients with $\mathrm{pT}$ class Tx were not included. 


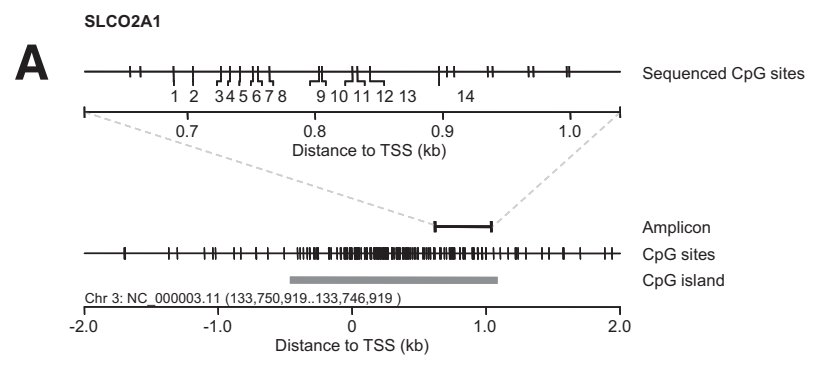

B
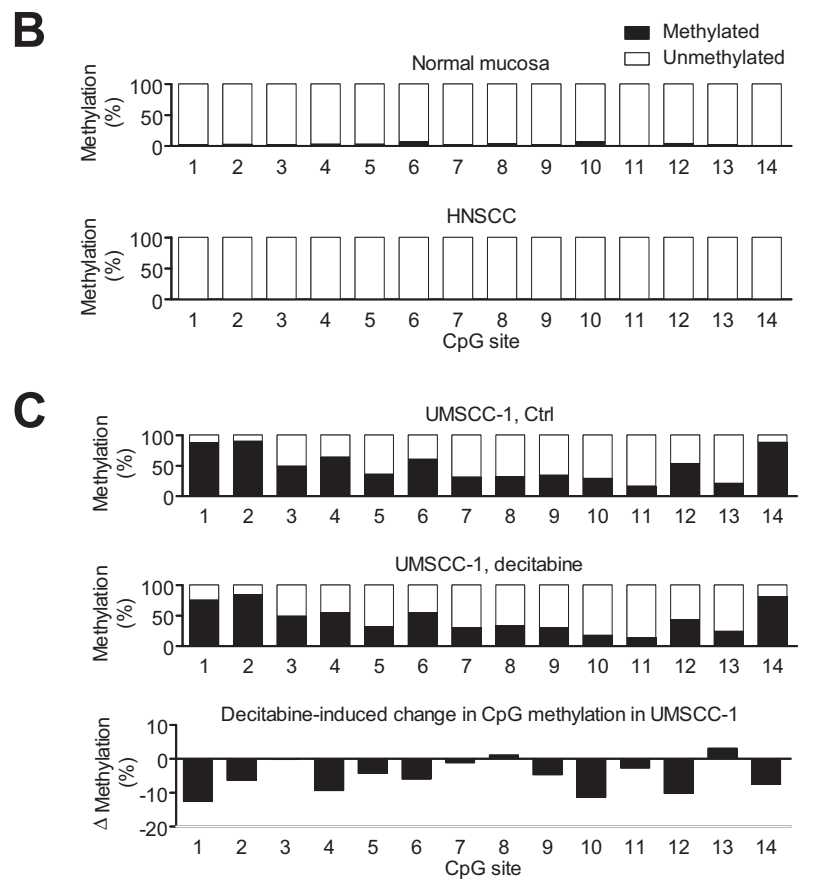

Figure 2 Analysis of $\mathrm{CpG}$ island methylation in SLCO2A1 using bisulfite sequencing. A: Position of the $\mathrm{CpG}$ island and the sequenced $\mathrm{CpG}$ sites relative to the transcription start site (TSS). B: Average methylation rate of individual $\mathrm{CpG}$ sites in $\mathrm{HNSCC}$ tumors and adjacent normal mucosa from two patients with HNSCC. C: Average methylation rate of individual CpG sites in vehicle-treated (Ctrl) and decitabine-treated $(1 \mu \mathrm{mol} / \mathrm{L}$ for 72 hours) UMSCC- 1 cells. Methylation rates of $26 \mathrm{CpG}$ sites were obtained by direct sequencing of PCR amplicons and by analysis of the sequence trace files using Epigenetic Sequencing Methylation Analysis Software.

transcription of $S L C O 2 B 1$, a gene that lacked $\mathrm{CpG}$ islands in its promoter region (defined as a DNA region of at least $200 \mathrm{bp}$ with a $\mathrm{C}+\mathrm{G}$ content of $50 \%$ and an observed $\mathrm{CpG}$ versus expected $\mathrm{CpG}$ in excess of 0.6). Moreover, decitabine treatment increased $A B C C 3$ transcript expression, although $\mathrm{CpG}$ islands in the $A B C C 3$ promoter were essentially unmethylated in both decitabine-treated and untreated cells (Figure 3). These findings suggested indirect effects of the DNA demethylating agent, decitabine, on transporter mRNA expression.

Because nuclear receptors, such as PXR, were capable of transcriptional activation, particularly of genes involved in drug metabolism and transport, and promoter methylation was involved in the regulation of NRII2/PXR in cancer cells, we hypothesized that nuclear receptors might play a role in the epigenetic regulation of transporter expression in HNSCC cells. Treatment of UMSCC-1 cells with decitabine induced a marked increase in NRII2/PXR (661-fold versus control; $P<0.001$ ) (Figure 5A). Qualitatively similar results were obtained when SCC-15 cells were investigated (12-fold increase versus control; $P<0.001$ ). These results supported the notion that the transcriptional regulator, $P X R$, was strongly induced by modification of the epigenome in HNSCC cells.

The activator of PXR, rifampicin, had no significant effect on SLCO2AI and $A B C C 3$ transcript levels (Figure 5B). This finding might be explained by the relatively low basal expression of NRII2/PXR in UMSCC-1 and SCC-15 cells. However, when NR1I2/PXR expression was enhanced by decitabine, cotreatment with rifampicin decreased transporter mRNA expression compared with cells treated with decitabine only (Figure 5B). For example, cotreatment of UMSCC-1 cells with decitabine and rifampicin reduced $S L C O 2 A 1$ and $A B C C 3$ mRNA expression by 78\% $(P<0.001)$ and 60\% $(P<0.001)$, respectively. These results supported a counterregulatory role of PXR on decitabine-induced transcription of SLCO2AI and ABCC3.

To further assess the role of NR1I2 on decitabine-induced increase in transporter mRNA expression, NRII2 expression

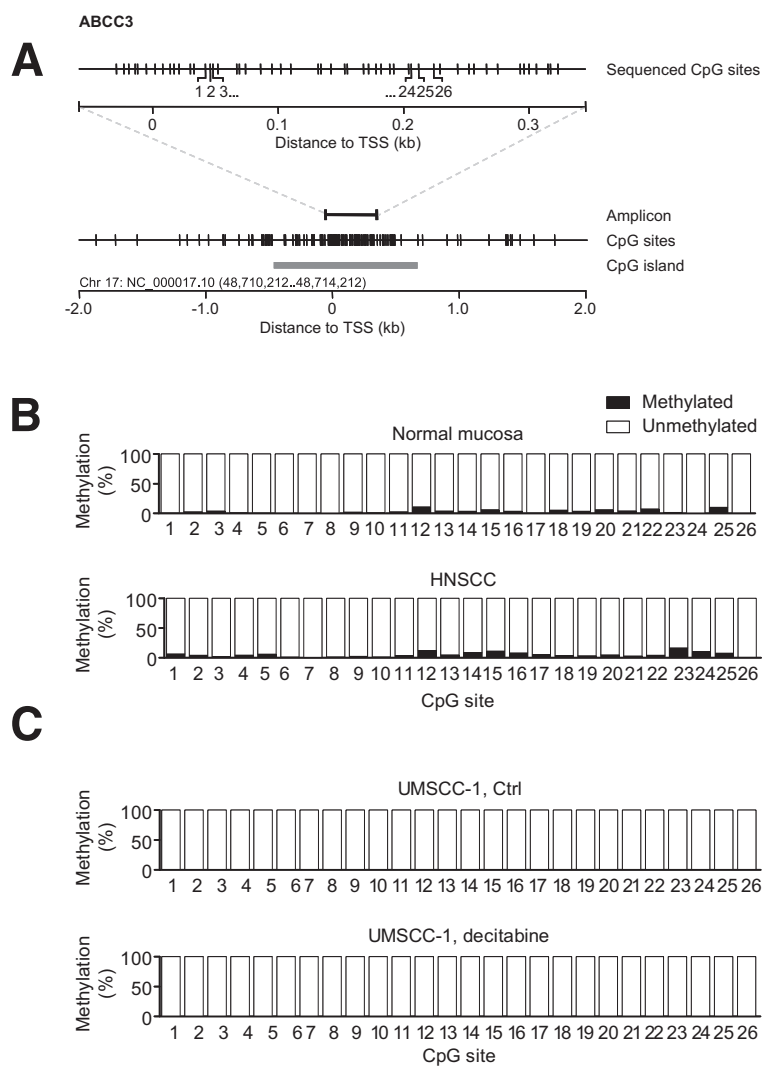

Figure 3 Analysis of $\mathrm{CpG}$ island methylation in $A B C C 3$ using bisulfite sequencing. A: Position of the $\mathrm{CpG}$ island and the sequenced $\mathrm{CpG}$ sites relative to the transcription start site (TSS). B: Average methylation rate of individual $\mathrm{CpG}$ sites in HNSCC tumors and adjacent normal mucosa from two patients with HNSCC. C: Average methylation rate of individual $\mathrm{CpG}$ sites in vehicle-treated (Ctrl) and decitabine-treated $(1 \mu \mathrm{mol} / \mathrm{L}$ for 72 hours) UMSCC- 1 cells. Methylation rates of $14 \mathrm{CpG}$ sites were obtained by direct sequencing of PCR amplicons and by analysis of the sequence trace files using Epigenetic Sequencing Methylation Analysis Software. 

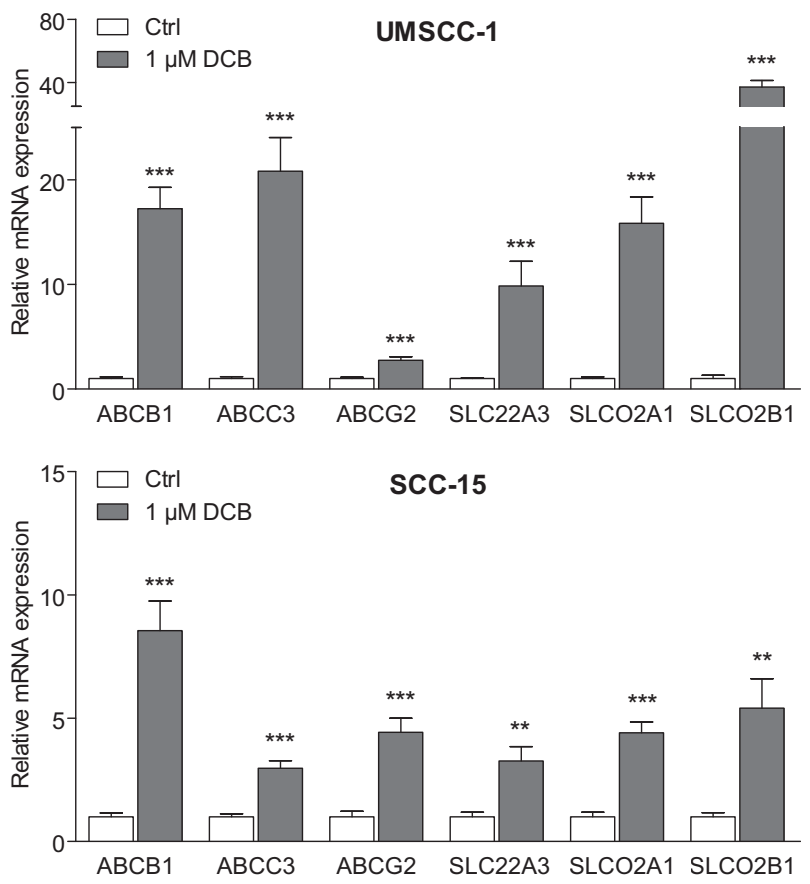

Figure 4 Effect of decitabine (DCB)-induced epigenetic modifications on the mRNA expression of selected $A B C, S L C 22$, and SLCO transporters in UMSCC- 1 and SCC-15 cells. Cells were treated with the DNA methyltransferase inhibitor, decitabine $(1 \mu \mathrm{mol} / \mathrm{L})$, for 72 hours. Control cells (Ctrl) were treated with solvent (dimethyl sulfoxide) only. Transporter mRNA expression was quantified by real-time RT-PCR and normalized to that of the reference genes $A C T B$ and GAPDH. mRNA expression in the drugtreated cells is presented relative to that in the solvent Ctrl. $n=11$ to 19 per group. ${ }^{* *} P<0.01,{ }^{* *} P<0.001$ versus Ctrl.

was stably silenced in UMSCC cells via infection with NRII2 shRNA lentiviruses. Two of four tested shRNA constructs silenced NRII2 mRNA expression lower than the detection limit (Figure 6A). In cells with effective NRII2 knockdown, decitabine treatment still resulted in an increase in $A B C C 3$ and $S L C O 2 A 1$ mRNA expression, but the effect was significantly reduced compared with untransfected cells [ABCC3, 6.7- versus 20.8-fold $(P<0.001)$; and SLCO2A1, 4.2- versus 15.8 -fold $(P<0.01)$ ], as shown in Figure 6B. Notably, in cells with NR1I2 knockdown, basal ABCC3 mRNA, but not $S L C O 2 A 1$ mRNA, levels were significantly reduced compared with parental cells, suggesting that basal ABCC 3 expression in UMSCC depended, in part, on NR1I2.

\section{Discussion}

This study demonstrates the following about expression of drug transporters: i) expression is markedly changed in the HNSCC tumor tissues compared with normal mucosa, ii) expression might be predictive of the outcome of patients with HNSCC, and iii) expression is affected by novel epigenetic therapies and is further modulated by nuclear receptor-mediated mechanisms.
To our knowledge, this is the first study that systematically compares gene expression profiles of membrane transporters, which are generally implicated in drug disposition or multidrug resistance, in human HNSCC tumors and adjacent normal mucosa. The most prominent changes in the expression profile were observed in transporters of the SLCO family, with an increased expression of SLCO1A2 and $S L C O 1 B 3$ and a decreased expression of $S L C O 2 B 1$, SLCO2A1, and SLCOICl in HNSCC tumor tissue compared with the normal mucosa.
A

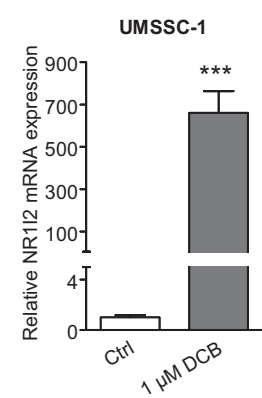

B
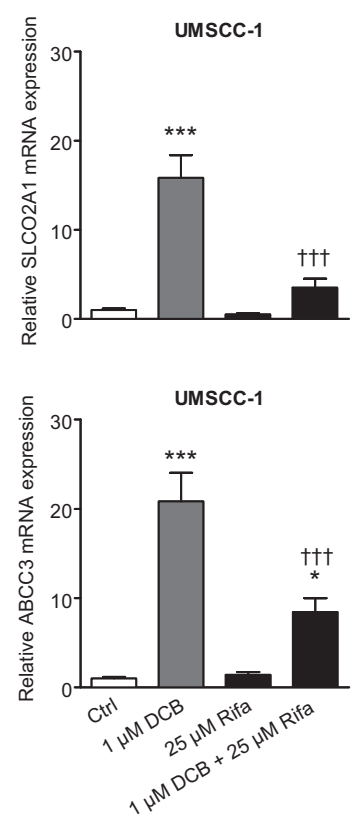

SCC-15
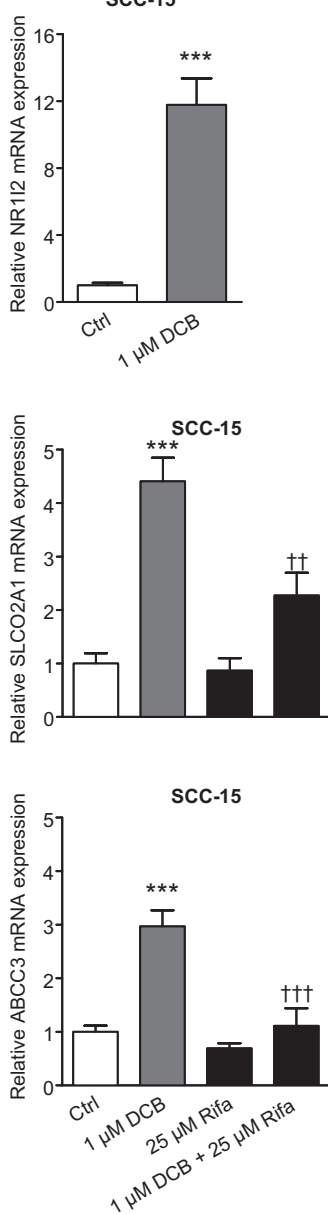

Figure 5 A: Effects of decitabine (DCB)-induced epigenetic modification on NR1I2/PXR mRNA expression in UMSCC-1 and SCC-15 cells. Cells were treated with $1 \mu \mathrm{mol} / L \mathrm{DCB}$ for 72 hours. Gene expression of NR1I2 was quantified by real-time RT-PCR and normalized to the expression of the reference genes $A C T B$ and GAPDH. mRNA expression in the drug-treated cells is presented relative to that in the solvent control cells (Ctrl). $n=$ 7 to 9 per group. B: Effects of the PXR ligand/activator rifampicin on SLCO2A1 and ABCC3 transcript expression in UMSCC-1 and SCC-15 cells, with or without concomitant $D C B$ treatment. Cells were treated with $D C B$ $(1 \mu \mathrm{mol} / \mathrm{L})$ and the PXR activator rifampicin $(25 \mu \mathrm{mol} / \mathrm{L})$, either alone or in combination, for 72 hours. Transporter gene expression was quantified by real-time RT-PCR and normalized to the expression of the reference genes $A C T B$ and GAPDH. mRNA expression in the drug-treated cells is presented relative to that in the solvent control cells (Ctrl). $n=7$ to 19 per group. ${ }^{*} P<0.05,{ }^{* * *} P<0.001$ versus $C \operatorname{trl} ;{ }^{\dagger \dagger} P<0.01,{ }^{\dagger \dagger \dagger} P<0.001$ versus $D C B$. 
A

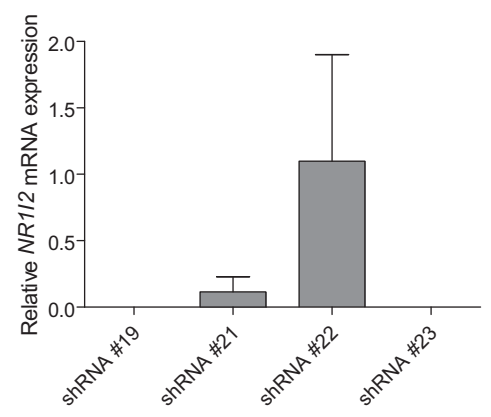

B
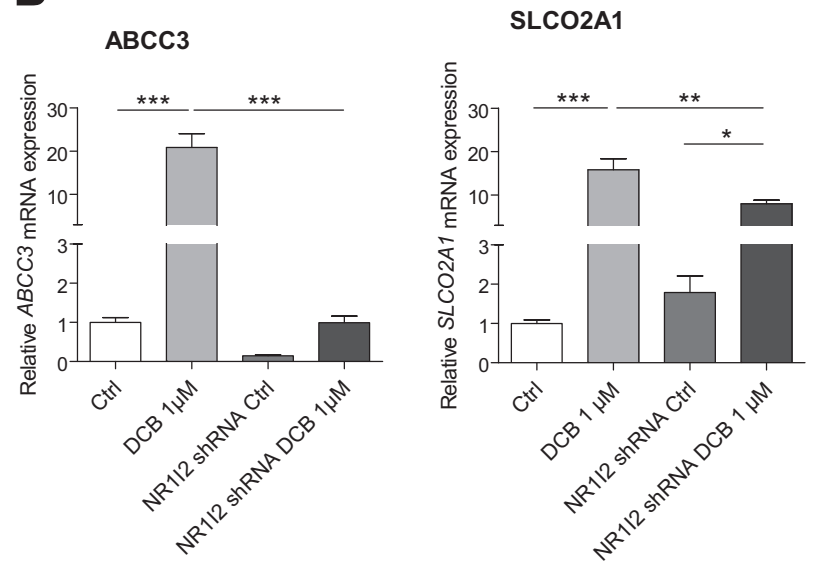

Figure 6 A: Quantitative assessment of the NR1I2/PXR knockdown efficiency. UMSCC cells were stably transfected with four different lentiviral NR1I2 shRNA constructs (number 19, TRCN00000211619; number 21, TRCN00000211621; number 22, TRCN00000211622; and number 23, TRCN00000211623), and NR1I2 mRNA expression was quantified by realtime RT-PCR and normalized to the expression of the reference genes $A C T B$ and GAPDH. mRNA levels are presented as fold change relative to control cells (Ctrl). $n=4$ independent measurements. B: Effect of NR1I2 silencing on SLCO2A1 and $A B C C 3$ transcript expression in UMSCC cells, with or without decitabine (DCB) treatment. Three UMSCC cell lines with efficient NR1I2/PXR knockdown (NR1I2 shRNA) were treated with $1 \mu \mathrm{mol} / \mathrm{L}$ decitabine for 72 hours. Transporter mRNA expression was quantified by real-time RT-PCR and normalized to the expression of the reference genes $A C T B$ and GAPDH. mRNA expression in the drug-treated cells is presented relative to that in the respective untreated $C$ trl. Pooled results from the three NR1I2 shRNA cell lines are shown. $n=4$ independent experiments. ${ }^{*} P<0.05,{ }^{* *} P<0.01$, and ${ }^{* *} P<0.001$.

Interestingly, a similar change in the expression of $S L C O 1 B 3$ and SLCO2A1 was found in colorectal cancer. $S L C O 1 B 3$ overexpression confers apoptotic resistance to colon cancer cells by altering p53-dependent pathways. ${ }^{24}$ SLCO2A1 can facilitate the removal of prostaglandin E2 from the extracellular milieu. In colorectal cancer, decreased SLCO2AI expression leads to higher concentrations of prostaglandin E2, which, in turn, mediates cell proliferation, antiapoptotic effects, and neovascularization. ${ }^{25}$ Taken together, these studies demonstrate that higher SLCO1B3 and lower SLCO2A1 expression in colorectal cancer provide a survival advantage to cancer cells. This also might hold true for HNSCC.

We observed a significant association between high $S L C O 2 B 1$ mRNA levels in HNSCC tumor tissues and a low
pT class and an improved 5-year survival of patients with HNSCC in a pilot study. In recent studies, expression levels of SLCO2B1 in liver, prostate, or breast cancer have been linked with patient outcomes, disease stage, or tumor differentiation. ${ }^{26-29}$ Based on these results, SLCO2B1 has been proposed as a biomarker for risk assessment. ${ }^{26-28}$ However, the specific mechanism by which SLCO2B1 affects patient outcome remains to be elucidated and most likely depends on the cancer type.

The SLCO2B1 expression level remained a significant predictor of 5-year survival in our study cohort, even when the pT class was considered as a covariate in a logistic regression analysis (odds ratio, 6.5; 95\% CI, 1.1 to 38.6; $P=0.04)$. This result suggests that $S L C O 2 B 1$ expression is linked to survival through additional mechanisms, irrespective of the tumor size. This might occur through the interaction of SLCO2B1 with the chemotherapeutic drugs. Patients in our study received a standard chemotherapy regimen for $\mathrm{HNSCC}^{30}$ that consisted of two or more of the following compounds: cisplatin, docetaxel, fluorouracil, and methotrexate. Although SLCO2B1 is a well-known uptake transporter for diverse drugs, such as statins, fexofenadine, glibenclamide, or montelukast, ${ }^{5,31}$ it is still unknown whether the standard chemotherapeutic drugs used in HNSCC treatment are substrates of SLCO2B1.

In our study, the transcript levels of $\mathrm{ABC}$ efflux transporters, which are commonly implicated in multidrug resistance, were unchanged $(A B C C 1, A B C C 2, A B C C 4, A B C C 5$, and $A B C C 10)$ or even reduced $(A B C C 3$ and $A B C G 2)$ in HNSCC tumor samples compared with adjacent normal mucosa. Moreover, our data did not reveal a significant association of $\mathrm{ABC}$ transporter expression in HNSCC tumor samples with 5 -year survival. Tsunoda et $\mathrm{al}^{32}$ found that $A B C G 2$ expression was an independent prognostic factor in esophageal squamous cell carcinoma. We were not able to replicate this finding in our cohort of patients with HNSCC; however, it is possible that the effects of $A B C G 2$ expression may have been missed because of the limited power of our study. None of the patients had received neoadjuvant chemotherapy before the tissue samples were obtained. We cannot rule out that the expression of $\mathrm{ABC}$ transporters may increase during chemotherapy because multidrug resistance often appears during drug treatment (secondary resistance), and that the $\mathrm{ABC}$ transporter levels at that stage may become predictive of outcome.

In the second part of our study, potential mechanisms that influence transporter expression were evaluated in cellular models of HNSCC. Modulation of the epigenome, particularly with the DNA methyltransferase inhibitor decitabine, markedly increased expression of some, but not all, investigated transporters of the $S L C O, S L C 22$, and $A B C$ families in HNSCC cells. Our results extend the findings of two previous studies ${ }^{33,34}$ that demonstrate the induction of $A B C G 2$ and SLCO1B3 in cancer cell lines by decitabine. The question of epigenetic modulation of transporter expression may have clinical implications because DNA 
methyltransferase inhibitors have already been approved as a treatment for lymphomas and are being tested as a therapeutic option against a variety of human solid tumors, including HNSCC. ${ }^{35-37}$ If these agents are concomitantly administered with classic anticancer drugs, the resultant induction of transporter expression may influence the disposition and effect of these anticancer drugs. ${ }^{9}$ In particular, enhanced expression of $\mathrm{ABCC} 3$ may induce drug resistance of cancer cells and affect efficacy of treatment with $\mathrm{ABCC} 3$ substrates, such as methotrexate, vincristine, doxorubicin, and cisplatin. On the other hand, decitabine-induced restoration of SLCO2A1 expression in tumor tissue may facilitate the removal from the extracellular milieu of prostaglandins, which were implicated as significant mediators in cancer progression.

Methylation of $\mathrm{CpG}$ islands in promoters of transporter genes was investigated in patient tissue samples and an HNSCC-derived tumor cell line. The UMSCC cell line had a different methylation signature than patient samples. Several studies ${ }^{38,39}$ demonstrated marked interpatient variability in promoter methylation at the single-gene level. Thus, the methylation status of the UMSCC cell line may reflect the high methylation status of the original HNSCC tumor sample. Another explanation for the discrepancy between the methylation status of the SLCO2Al promoter in the HNSCC tissue and the UMSCC cell line is that cell line methylation is affected by the tissue culture environment.

An important finding of our study was that the presence of $\mathrm{CpG}$ islands in the promoters of transporter genes was generally not required for decitabine-mediated induction of their expression. Inhibition of DNA methyltransferase, for example, caused a substantial increase (37-fold) in the transcript levels of $S L C O 2 B 1$, a gene that completely lacks $\mathrm{CpG}$ islands in its promoter region. Although a small decrease in the promoter $\mathrm{CpG}$ methylation of SLCO2Al after decitabine treatment partially explains the induction of SLCO2A1, no correlation between $A B C C 3$ promoter methylation and induction of $A B C C 3$ mRNA was observed.

Decitabine targets the whole epigenome, including the epigenetic state of transcriptional regulators, such as NRII2/ $P X R$. Given that PXR is capable of transcriptional activation of genes involved in drug transport, such as $A B C B 1,{ }^{40}$ and promoter methylation is involved in the regulation of NRII2/PXR in cancer cells, ${ }^{16,17}$ we hypothesized that nuclear receptors may participate in the epigenetic regulation of transporter expression in HNSCC cells. Consistent with a previous report on colon carcinoma, ${ }^{16}$ decitabine markedly induced NRII2/PXR in UMSCC-1 and SCC-15 cell lines. Furthermore, NR1I2/PXR knockdown significantly attenuated the inducing effects of decitabine on $A B C C 3$ and SLCO2A1 mRNA expression. Surprisingly, cotreatment of these cells with a prototypical PXR activator caused a significant repression of SLCO2AI and ABCC3 compared with cells treated with decitabine alone. This finding partially corresponds to observations made by Cheng et $\mathrm{al}^{41}{ }^{41}$ who observed a down-regulation of several
Slcos, including Slco2al, in the mouse liver after exposure to prototypical PXR ligands. Several anticancer drugs, such as cisplatin, paclitaxel, and cyclophosphamide, are wellknown agonists of nuclear receptors. ${ }^{14,15,42}$ This raises the possibility of drug-drug interactions in patients treated with combinations of multiple anticancer drugs, which, in turn, may affect the treatment outcome. ${ }^{43}$ Taken together, our results demonstrate an interaction between epigenetic and nuclear receptor-mediated mechanisms in the regulation of transporter expression.

To our knowledge, this is the first study to provide a basis for the assessment of the role of uptake and efflux transporters in the accessibility of HNSCC tumors to chemotherapeutic agents. Transporters that are differentially expressed in HNSCC tumors versus normal mucosa were identified. Moreover, the correlation of SLCO2B1 levels in HNSCC tumors with 5-year survival of patients was demonstrated in a pilot study. Replication of these results in a prospective observational study is necessary. Modulation of the epigenome by the DNA methyltransferase inhibitor, decitabine, induced the expression of several transporters considerably, suggesting the involvement of epigenetic mechanisms in the regulation of transporter expression. These mechanisms do not necessarily involve direct hypomethylation of $\mathrm{CpG}$ islands in transporter genes, and the pathway by which transporter mRNA levels (eg, those of $S L C O 2 B 1$ and $A B C C 3$ ) are induced by decitabine remains to be elucidated. Finally, interactions between epigenetic and nuclear receptor-mediated mechanisms played a role in the regulation of transporter expression.

\section{References}

1. Huang Y: Pharmacogenetics/genomics of membrane transporters in cancer chemotherapy. Cancer Metastasis Rev 2007, 26:183-201

2. Zolk O: Disposition of metformin: variability due to polymorphisms of organic cation transporters. Ann Med 2012, 44:119-129

3. Szakacs G, Paterson JK, Ludwig JA, Booth-Genthe C, Gottesman MM: Targeting multidrug resistance in cancer. Nat Rev Drug Discov 2006, 5:219-234

4. Solbach TF, König J, Fromm MF, Zolk O: ATP-binding cassette transporters in the heart. Trends Cardiovasc Med 2006, 16:7-15

5. Fahrmayr C, Fromm MF, König J: Hepatic OATP and OCT uptake transporters: their role for drug-drug interactions and pharmacogenetic aspects. Drug Metab Rev 2010, 42:380-401

6. König J, Seithel A, Gradhand U, Fromm MF: Pharmacogenomics of human OATP transporters. Naunyn Schmiedebergs Arch Pharmacol 2006, 372:432-443

7. Hagenbuch B, Gui C: Xenobiotic transporters of the human organic anion transporting polypeptides (OATP) family. Xenobiotica 2008, 38 : 778-801

8. Ferlay J, Shin HR, Bray F, Forman D, Mathers C, Parkin DM: Estimates of worldwide burden of cancer in 2008: GLOBOCAN 2008. Int J Cancer 2010, 127:2893-2917

9. Stewart DJ, Issa JP, Kurzrock R, Nunez MI, Jelinek J, Hong D, Oki Y, Guo Z, Gupta S, Wistuba II: Decitabine effect on tumor global DNA methylation and other parameters in a phase I trial in refractory solid tumors and lymphomas. Clin Cancer Res 2009, 15:3881-3888

10. Taberlay PC, Jones PA: DNA methylation and cancer. Prog Drug Res 2011, 67:1-23 
11. Bram EE, Stark M, Raz S, Assaraf YG: Chemotherapeutic druginduced $\mathrm{ABCG} 2$ promoter demethylation as a novel mechanism of acquired multidrug resistance. Neoplasia 2009, 11:1359-1370

12. Bennett KL, Karpenko M, Lin MT, Claus R, Arab K, Dyckhoff G, Plinkert P, Herpel E, Smiraglia D, Plass C: Frequently methylated tumor suppressor genes in head and neck squamous cell carcinoma. Cancer Res 2008, 68:4494-4499

13. Tirona RG: Molecular mechanisms of drug transporter regulation. Handb Exp Pharmacol 2011, (201):373-402

14. Harmsen S, Meijerman I, Febus CL, Maas-Bakker RF, Beijnen JH, Schellens JH: PXR-mediated induction of P-glycoprotein by anticancer drugs in a human colon adenocarcinoma-derived cell line. Cancer Chemother Pharmacol 2010, 66:765-771

15. Masuyama H, Suwaki N, Tateishi Y, Nakatsukasa H, Segawa T, Hiramatsu $\mathrm{Y}$ : The pregnane $\mathrm{X}$ receptor regulates gene expression in a ligand- and promoter-selective fashion. Mol Endocrinol 2005, 19: $1170-1180$

16. Habano W, Gamo T, Terashima J, Sugai T, Otsuka K, Wakabayashi G, Ozawa S: Involvement of promoter methylation in the regulation of Pregnane X receptor in colon cancer cells. BMC Cancer 2011, 11:81

17. Misawa A, Inoue J, Sugino Y, Hosoi H, Sugimoto T, Hosoda F, Ohki M, Imoto I, Inazawa J: Methylation-associated silencing of the nuclear receptor $1 \mathrm{I} 2$ gene in advanced-type neuroblastomas, identified by bacterial artificial chromosome array-based methylated $\mathrm{CpG}$ island amplification. Cancer Res 2005, 65:10233-10242

18. Krause CJ, Carey TE, Ott RW, Hurbis C, McClatchey KD, Regezi JA: Human squamous cell carcinoma: establishment and characterization of new permanent cell lines. Arch Otolaryngol 1981, 107:703-710

19. Brenner JC, Graham MP, Kumar B, Saunders LM, Kupfer R, Lyons RH, Bradford CR, Carey TE: Genotyping of 73 UM-SCC head and neck squamous cell carcinoma cell lines. Head Neck 2010, 32: 417-426

20. Zhao M, Sano D, Pickering CR, Jasser SA, Henderson YC, Clayman GL, Sturgis EM, Ow TJ, Lotan R, Carey TE, Sacks PG, Grandis JR, Sidransky D, Heldin NE, Myers JN: Assembly and initial characterization of a panel of 85 genomically validated cell lines from diverse head and neck tumor sites. Clin Cancer Res 2011, 17:7248-7264

21. Eckhardt F, Lewin J, Cortese R, Rakyan VK, Attwood J, Burger M, Burton J, Cox TV, Davies R, Down TA, Haefliger C, Horton R, Howe K, Jackson DK, Kunde J, Koenig C, Liddle J, Niblett D, Otto T, Pettett R, Seemann S, Thompson C, West T, Rogers J, Olek A, Berlin K, Beck S: DNA methylation profiling of human chromosomes 6, 20 and 22. Nat Genet 2006, 38:1378-1385

22. Lewin J, Schmitt AO, Adorjan P, Hildmann T, Piepenbrock C: Quantitative DNA methylation analysis based on four-dye trace data from direct sequencing of PCR amplificates. Bioinformatics 2004, 20:3005-3012

23. Stefanska B, Huang J, Bhattacharyya B, Suderman M, Hallett M, Han ZG, Szyf M: Definition of the landscape of promoter DNA hypomethylation in liver cancer. Cancer Res 2011, 71:5891-5903

24. Lee W, Belkhiri A, Lockhart AC, Merchant N, Glaeser H, Harris EI, Washington MK, Brunt EM, Zaika A, Kim RB, El-Rifai W: Overexpression of OATP1B3 confers apoptotic resistance in colon cancer. Cancer Res 2008, 68:10315-10323

25. Holla VR, Backlund MG, Yang P, Newman RA, DuBois RN: Regulation of prostaglandin transporters in colorectal neoplasia. Cancer Prev Res (Phila) 2008, 1:93-99

26. Pressler H, Sissung TM, Venzon D, Price DK, Figg WD: Expression of OATP family members in hormone-related cancers: potential markers of progression. PLoS One 2011, 6:e20372

27. Yang M, Xie W, Mostaghel E, Nakabayashi M, Werner L, Sun T, Pomerantz M, Freedman M, Ross R, Regan M, Sharifi N, Figg WD, Balk S, Brown M, Taplin ME, Oh WK, Lee GS, Kantoff PW: SLCO2B1 and SLCO1B3 may determine time to progression for patients receiving androgen deprivation therapy for prostate cancer. J Clin Oncol 2011, 29:2565-2573

28. Wright JL, Kwon EM, Ostrander EA, Montgomery RB, Lin DW, Vessella R, Stanford JL, Mostaghel EA: Expression of SLCO transport genes in castration-resistant prostate cancer and impact of genetic variation in SLCO1B3 and SLCO2B1 on prostate cancer outcomes. Cancer Epidemiol Biomarkers Prev 2011, 20:619-627

29. Al Sarakbi W, Mokbel R, Salhab M, Jiang WG, Reed MJ, Mokbel K: The role of STS and OATP-B mRNA expression in predicting the clinical outcome in human breast cancer. Anticancer Res 2006, 26: 4985-4990

30. Furness S, Glenny AM, Worthington HV, Pavitt S, Oliver R, Clarkson JE, Macluskey M, Chan KK, Conway DI: Interventions for the treatment of oral cavity and oropharyngeal cancer: chemotherapy. Cochrane Database Syst Rev 2011, (4):CD006386

31. Anderson CM, Thwaites DT: Hijacking solute carriers for protoncoupled drug transport. Physiology (Bethesda) 2010, 25:364-377

32. Tsunoda S, Okumura $\mathrm{T}$, Ito $\mathrm{T}$, Kondo $\mathrm{K}$, Ortiz $\mathrm{C}$, Tanaka $\mathrm{E}$, Watanabe G, Itami A, Sakai Y, Shimada Y: ABCG2 expression is an independent unfavorable prognostic factor in esophageal squamous cell carcinoma. Oncology 2006, 71:251-258

33. Ichihara S, Kikuchi R, Kusuhara H, Imai S, Maeda K, Sugiyama Y: DNA methylation profiles of organic anion transporting polypeptide 1B3 in cancer cell lines. Pharm Res 2010, 27:510-516

34. To KK, Zhan Z, Bates SE: Aberrant promoter methylation of the ABCG2 gene in renal carcinoma. Mol Cell Biol 2006, 26: $8572-8585$

35. Abele R, Clavel M, Dodion P, Bruntsch U, Gundersen S, Smyth J, Renard J, van Glabbeke M, Pinedo HM: The EORTC Early Clinical Trials Cooperative Group experience with 5-aza-2'-deoxycytidine (NSC 127716) in patients with colo-rectal, head and neck, renal carcinomas and malignant melanomas. Eur J Cancer Clin Oncol 1987, 23:1921-1924

36. Kim J, Guan J, Chang I, Chen X, Han D, Wang CY: PS-341 and histone deacetylase inhibitor synergistically induce apoptosis in head and neck squamous cell carcinoma cells. Mol Cancer Ther 2010, 9: 1977-1984

37. Prystowsky MB, Adomako A, Smith RV, Kawachi N, McKimpson W, Atadja P, Chen Q, Schlecht NF, Parish JL, Childs G, Belbin TJ: The histone deacetylase inhibitor LBH589 inhibits expression of mitotic genes causing G2/M arrest and cell death in head and neck squamous cell carcinoma cell lines. J Pathol 2009, 218:467-477

38. Dong SW, Zhang P, Liu YM, Cui YT, Wang S, Liang SJ, He Z, Sun P, Wang YG: Study on RIZ1 gene promoter methylation status in human esophageal squamous cell carcinoma. World J Gastroenterol 2012, 18: $576-582$

39. Hanada N, Takahata T, Zhou Q, Ye X, Sun R, Itoh J, Ishiguro A, Kijima H, Mimura J, Itoh K, Fukuda S, Saijo Y: Methylation of the KEAP1 gene promoter region in human colorectal cancer. BMC Cancer 2012, 12:66

40. Geick A, Eichelbaum M, Burk O: Nuclear receptor response elements mediate induction of intestinal MDR1 by rifampin. J Biol Chem 2001, 276:14581-14587

41. Cheng X, Maher J, Dieter MZ, Klaassen CD: Regulation of mouse organic anion-transporting polypeptides (Oatps) in liver by prototypical microsomal enzyme inducers that activate distinct transcription factor pathways. Drug Metab Dispos 2005, 33:1276-1282

42. Synold TW, Dussault I, Forman BM: The orphan nuclear receptor SXR coordinately regulates drug metabolism and efflux. Nat Med 2001, 7:584-590

43. Harmsen S, Meijerman I, Beijnen JH, Schellens JH: The role of nuclear receptors in pharmacokinetic drug-drug interactions in oncology. Cancer Treat Rev 2007, 33:369-380 